\title{
Phenotypic Characterization of Mutants from Different Species of Annual Chrysanthemum
}

\author{
Renu* and Shant Lal \\ Department of Horticulture, G. B. Pant University of Agriculture \& Technology \\ Pantnagar-263145 District: Udham Singh Nagar, Uttarakhand, India \\ *Corresponding author
}

\begin{abstract}
A B S T R A C T
Keywords

Phenotypic

Characterization;

Chrysanthemum

coronarium;

Chrysanthemum

segetum;

Chrysanthemum carinatum; mutants

Article Info

Accepted:

15 August 2019

Available Online:

10 September 2019

An experiment was conducted to study the phenotypic characterization of mutants from different species of annual chrysanthemum by treating the seeds of different species of Chrysanthemum coronarium (Cco), Chrysanthemum segetum (Cse) and Chrysanthemum carinatum (Cca) with various doses of gamma rays (Cobalt-60) at National Botanical Research Institute, Lucknow. Immediately after the mutagenic treatment, the nurseries were raised and further transplanted in the field. $\mathbf{M}_{1}$ population of all three species of Chrysanthemum treated with different doses of gamma rays were screened and characterized. The experimental materials selected for the present investigation consisted of 22 mutant $\left(\mathrm{M}_{2}\right)$ lines of species Chrysanthemum coronarium (Cco), 5 mutant lines of species Chrysanthemum segetum (Cse) and 3 mutant lines of species Chrysanthemum carinatum (Cca).Data on phenotypic characterization conducted for various vegetative and floral traits revealed that there is a significant variation among different mutants and all three species of Chrysanthemum used for the study of various phenotypic parameters. Result of quantitative analysis revealed thatin case of species Chrysanthemum coronarium, mutant $\mathrm{Co}_{3-63}$ had maximum leaf length, flower head weight and number of ray florets whereas leaf width and leaf area was found maximum in mutant Co 11-131. Mutant Co 2-5 and mutant $\mathrm{Co}_{5-55}$ showed maximum flower head diameter, ray floret weight and ray floret length, ray floret width respectively. However, mutant $\mathrm{Co}{ }_{11-56}$ had maximum flower head weight. In species Chrysanthemum segetum, mutant $\mathrm{S}_{4-9}$ had maximum flower head diameter and disc floret weight whereas mutant $\mathrm{S}_{4-6}$ had maximum ray floret weight and a greater number of disc florets. Among the flowering characters like flower head height, number of flowers per plant and flower head weight, mutants, $S_{1-14}$ and $S_{1-5}$ performed well. In the species Chrysanthemum carinatum, mutant $\mathrm{C}_{13-1}$ showed the best results with respect to vegetative and flowering characters as it had maximum leaf width, leaf area, number of ray florets, flower head weight and flower head height. However, mutant $\mathrm{C}_{15-1}$ had maximum ray floret weight.
\end{abstract}

\section{Introduction}

Among the flowers, Chrysanthemum (chrysos means 'golden' and anthos means 'flower') is a popular flower crop of commercial importance. Chrysanthemum belongs to family Asteraceae also called the aster, daisy, or sunflower family native to the northern 
hemisphere, chiefly Europe and Asia. It has approximately 1,620 genera and more than 23,600 species (Stevens, 2001). In India, 900 species in 167 genera (Hajra et al., 1995) are reported. The family is further subdivided into 12 subfamilies, dominated by the large subfamily asteroideae that contains more than 70 per cent of the species of the family. It is an important global floriculture crop leader due to its unparalleled diversity in plant and flower colour, shape, form and blooming pattern. In international florists' trade, Chrysanthemum ranks third as a cut flower and fifth as pot plant (Anonymous, 2008). It is characterized by annual or perennial herb or woody nature. In the present study, all three species of Chrysanthemum are annual and strictly cross pollinated due to self-incompatibility. Selfing is generally not possible, although some pseudo self-incompatible plants have been discovered (Anderson et al., 1992). The rate of successful crosses between related and unrelated cultivars is low, usually only 5 per cent to 50 per cent (Zagorski et al., 1983). Chrysanthemum coronarium (crown daisy, garland Chrysanthemum or edible Chrysanthemum) commonest of all annual Chrysanthemum s. Capitula are solitary with creamy white florets having yellowish tinged at the base, usually not in corymbs. Chrysanthemum segetum also called Corn marigold is native to Eastern Mediterranean and North Africa. It is easily distinguished by greyish green toothed leaves, stem simple to somewhat branched. Capitula are solitary with golden yellow florets, usually not in corymbs. However, Chrysanthemum carinatum also called tricolored Chrysanthemum or keeled Chrysanthemum is native to Morocco (North Africa). It is the commonest and gaudiest of all annual Chrysanthemum s, easily distinguished by keeled or ridges scale (carinatum means keeled) of the involucres and dark purple disc. Capitula are solitary with white florets having a yellow ring at the base, in corymb.
In floriculture industry, there is constant demand for novelty in existing crops. Development of new cultivars through conventional or modern techniques have been a prime objective in commercial floriculture. New color, earliness, stem length, number of flowers, plant architecture, resistance to abiotic and biotic stresses, productivity and vase life are the main attributes required in new cultivars. These new cultivars in existing crops could be produced by the introduction, hybridization and through molecular techniques. Over the past 50 years, the use of induced mutation through irradiation and chemical agents have also played a major role in the development of superior crop varieties. Induced mutagenesis in Chrysanthemum is the most successful story. Mutants are phenotypically different from parental variety only in flower color/shape and leaf variegation (Datta, 1988). New ornamental plant varieties are continuously being created by breeders in response to consumer demand for new products. Any change in the dominant genes is easily expressed in the first generation and thus the selection of mutant of directly perceptible characters like flower colour, shape, size and large number of new flower color/type, chlorophyll variegated mutant varieties have been developed worldwide and commercialized.

\section{Materials and Methods}

The present investigation entitled "Phenotypic Characterization of Mutants from Different Species of Annual Chrysanthemum "was conducted at Model Floriculture Centre, Department of Horticulture in G. B. Pant University of Agriculture and Technology, Pantnagar, Uttarakhand. The region is characterized by humid subtropical climate with the maximum temperature ranging from $30^{\circ} \mathrm{C}$ to $45^{\circ} \mathrm{C}$ in summer and minimum $3.7^{\circ} \mathrm{C}$ to $12.9^{\circ} \mathrm{C}$ in winter. The experimental materials for the present investigation comprised of three species of Chrysanthemum 
and their different mutants (Table A). $\mathrm{M}_{1}$ population of all three species of Chrysanthemum treated with different doses of gamma rays at National Botanical Research Institute, Lucknow were screened and characterized. The list of various gamma rays doses and their duration of exposure are given in (Table B).

\section{Experimental design}

\section{Layout of experiment}

Randomized Block Design (RBD)

Replications $\quad-\quad 3$

Number of species $\quad-\quad 3$

Number of selected mutants - 30

1. C. coronarium : 22
2. C. segetum: 5

3. C. carinatum : 3

Total treatment $-30+3$ genotype $=33$

Planting distance $-50 \times 30 \mathrm{~cm}$

Date of Planting - September

Date of Transplanting - October (30 days old seedling).

\section{Experimental methods}

The experiment broadly consisted of two parts:

1. Phenotypic characterization of mutants and their parents of three Chrysanthemum species.

2. Identification of desirable mutants.

Table.A Planting material

\begin{tabular}{|l|l|l|}
\hline S. No. & Genotype & Screened Mutants $\left(\mathbf{M}_{2}\right)$ lines \\
\hline $\mathbf{1}$ & Chrysanthemum coronarium & $\begin{array}{l}\mathrm{Co}_{1-9}, \mathrm{Co}_{2-5}, \mathrm{Co}_{3-1}, \mathrm{Co}_{3-59}, \mathrm{Co}_{3-63}, \mathrm{Co}_{4-1}, \mathrm{Co}_{4-67}, \mathrm{Co}_{4-88,}, \\
\mathrm{Co}_{5-55}, \mathrm{Co}_{7-16}, \mathrm{Co}_{7-88}, \mathrm{Co}_{8-5}, \mathrm{Co}_{8-11}, \mathrm{Co}_{10-24}, \mathrm{Co}_{10-26}, \mathrm{Co}_{11-56}, \\
\mathrm{Co}_{11-131}, \mathrm{Co}_{12-3}, \mathrm{Co}_{12-97}, \mathrm{Co}_{13-7}, \mathrm{Co}_{13-23}, \mathrm{Co}_{13-45}\end{array}$ \\
\hline $\mathbf{2}$ & Chrysanthemum segetum & $\mathrm{S}_{1-5}, \mathrm{~S}_{1-14}, \mathrm{~S}_{4-6}, \mathrm{~S}_{4-9}, \mathrm{~S}_{5-4}$ \\
\hline $\mathbf{3}$ & Chrysanthemum carinatum & $\mathrm{C}_{9-1}, \mathrm{C}_{13-1}, \mathrm{C}_{15-1}$ \\
\hline
\end{tabular}

Table.B Doses and duration of gamma rays used with different species of Chrysanthemum

\begin{tabular}{|c|c|c|c|}
\hline S. No. & Symbol used & Dose (KR) & Duration of exposure \\
\hline $\mathbf{1}$ & $\mathrm{T}_{0}$ & 0 (control) & 0 \\
\hline $\mathbf{2}$ & $\mathrm{T}_{1}$ & 2 & $1 \mathrm{~min} 20 \mathrm{sec}$ \\
\hline $\mathbf{3}$ & $\mathrm{T}_{2}$ & 4 & $2 \mathrm{~min} 40 \mathrm{sec}$ \\
\hline $\mathbf{4}$ & $\mathrm{T}_{3}$ & 6 & $4 \mathrm{~min}$ \\
\hline $\mathbf{5}$ & $\mathrm{T}_{4}$ & 8 & $5 \mathrm{~min} 20 \mathrm{sec}$ \\
\hline $\mathbf{6}$ & $\mathrm{T}_{5}$ & 10 & $6 \mathrm{~min} 40 \mathrm{sec}$ \\
\hline
\end{tabular}

\section{Results and Discussion}

\section{Chrysanthemum coronarium}

It is evident from the data presented in Table 1and 2 that there is a significant effect of vegetative and flowering characteristics of species Chrysanthemum coronarium $(\mathrm{Cco})$ and its all mutants. Among all the characters plant height $(120.73 \mathrm{~cm})$, plant spread (E-W) $(75.73$ $\mathrm{cm})$ and $(\mathrm{N}-\mathrm{S})(73.52 \mathrm{~cm})($ Fig 1), No. of 
flowers per plant (46.68 no.), No. of disc florets (472.10 no.) and disc floret weight (2.34 mg) (Fig 2)were found maximum in species C. coronarium (Cco) while, mutant $\mathrm{Co}_{3-63}$ had greater leaf length $(6.60 \mathrm{~cm})$, flower head height $(4.23 \mathrm{~cm})$ and number of ray florets (453.41 no) and mutant $\mathrm{Co}_{2-5}$ showed maximum flower head diameter $(7.87 \mathrm{~cm})$ and ray floret weight $(34.81 \mathrm{mg})$. Reduction in plant height of all the mutants than control is due to the inactivation of auxin content with increase in radiation dose which also depend on nature and extent of chromosomal damage (Banerji and Datta, 2002). The maximum leaf area was found in mutant Co $11-131\left(12.27 \mathrm{~cm}^{2}\right)$ which was statistically at par with mutant Co $4-88\left(11.97 \mathrm{~cm}^{2}\right)$ and significantly higher than others while the minimum leaf area was found in species Cco $\left(7.13 \mathrm{~cm}^{2}\right)$. The maximum leaf length was found in mutant $\mathrm{Co}_{3-63}(6.60 \mathrm{~cm})$ which was statistically at par with mutants, $\mathrm{Co}_{8-11}, \mathrm{Co}_{11-131}, \mathrm{Cco}, \mathrm{Co}_{1-9}$ and mutant $\mathrm{Co}_{3-59}$ and significantly higher than other mutants while leaf length was observed minimum $(3.46 \mathrm{~cm})$ in mutant $\mathrm{Co}_{12-3}$. Reduction of plant growth causes reduction of leaf length which may be due to the physiological, morphological and cytological disturbance caused by gamma radiation (Gaul, 1970). Ray floret length $(3.47 \mathrm{~cm})$ and width $(1.43 \mathrm{~cm})$ was found more in mutant Co 5-55 whereas mutant $\mathrm{Co}_{11-56}$ had maximum flower head weight $(4.96 \mathrm{~g})$. These findings were in close agreement of Banerji and Datta (1990)and Furuya (1998) in Chrysanthemum.

\section{Chrysanthemum segetum}

Significant variation in different quantitative traits which represented in Table 3and 4 revealed that the maximum plant height (75.59 $\mathrm{cm})$, plant spread $(\mathrm{E}-\mathrm{W})(66.62 \mathrm{~cm})$ and $(\mathrm{N}-\mathrm{S})$ $(65.40 \mathrm{~cm})$, flower disc diameter $(2.43 \mathrm{~cm})$, leaf length $(6.95 \mathrm{~cm})$, ray floret length $(2.81$ $\mathrm{cm})$ and ray floret width $(1.40 \mathrm{~cm})$ were found in species $C$. segetum(Cse) while, minimum plant height $(42.86 \mathrm{~cm})$, plant spread $(\mathrm{E}-\mathrm{W})$ $(40.71 \mathrm{~cm})$ and $(\mathrm{N}-\mathrm{S})(37.38 \mathrm{~cm})($ Fig 3$)$ was found in mutant $\mathrm{S}_{4-6}$ lesser plant spread depicts less number of branches per plant, which may be due to high mutagenic inhibitory effect of gamma rays. Misra et al., (2009) also noted reduction in plant vigor after gamma rays treatment. Mutant $\mathrm{S}_{5-4}$ showed the minimum leaf length $(4.76 \mathrm{~cm})$ however, minimum ray floret length was noted in mutant $\mathrm{S}_{1-5}$ (1.98 $\mathrm{cm})$ and mutants $\mathrm{S}_{4-6}$ and $\mathrm{S}_{4-9}$ observed minimum ray floret width $(0.67 \mathrm{~cm})$. Ray floret weight $(25.46 \mathrm{mg})$ was noted higher in mutant $\mathrm{S}_{4-6}$ while minimum in mutant $\mathrm{S}_{5-4}$ (23.55 mg) (Fig 4). The maximum number of flowers/plants was found in mutant $\mathrm{S}_{1-14}$ (65.73 no) while, minimum number of flowers/plants was found in mutant $\mathrm{S}_{5-4}(41.85$ no).Mutant $\mathrm{S}_{4-9}$ hadmaximum flower head diameter $(7.10 \mathrm{~cm})$ which was statistically at par with mutant $\mathrm{S}_{4-6}(6.84 \mathrm{~cm})$ and significantly higher than other mutants while, minimum flower head diameter was found in mutant $\mathrm{S}_{1-5}(5.26 \mathrm{~cm})$ these findings were in the line of agreement as reported by Banerji and Datta (1990) in Chrysanthemum . They observed the significant reduction in survival, number of branches, leaves and flower head size as compared to the control. Number of disc florets (499.25 no) was noted higher in mutant $\mathrm{S}_{4-6}$ which was statistically at par with mutant $\mathrm{S}_{4-9}$ (457.42 no)and minimum number of disc florets was found in mutant $\mathrm{S}_{5-4}$ (351.70 no). Mutant $S_{1-5}$ observed a maximum flower head weight $(2.36 \mathrm{~g})$ which was statistically at par with mutants $\mathrm{S}_{1-14}(2.32$ $\mathrm{g}), \mathrm{S}_{4-9}(2.33 \mathrm{~g})$ and $\mathrm{S}_{4-6}$ and species Cse $(2.19 \mathrm{~g})$ whereas, minimum flower head weight was found in mutant $\mathrm{S}_{5-4}(21.95$ g).These findings were in close confirmation of Barakat et al., (2010) in Chrysanthemum who observed that the irradiation dose $0.5 \mathrm{~Gy}$ was found the most effective dose for inducing mutation in flower shape, number of florets per flower head and conversion from tubular florets to spoon shaped florets. 
Table.1 Phenotypic characterization of $C$. coronarium and its different mutants

\begin{tabular}{|c|c|c|c|c|c|c|c|c|}
\hline Species & $\begin{array}{l}\text { Plant } \\
\text { height } \\
\text { (cm) }\end{array}$ & $\begin{array}{l}\text { Plant spread } \\
(\text { E-W })(\mathrm{cm})\end{array}$ & $\begin{array}{l}\text { Plant spread } \\
(\mathrm{N}-\mathrm{S})(\mathrm{cm})\end{array}$ & $\begin{array}{l}\text { Leaf length } \\
(\mathrm{cm})\end{array}$ & $\begin{array}{l}\text { Leaf } \\
\text { width } \\
\text { (cm) }\end{array}$ & $\begin{array}{l}\text { Leaf area } \\
\left(\mathrm{cm}^{2}\right)\end{array}$ & $\begin{array}{l}\text { No of } \\
\text { flowers/ } \\
\text { plant }\end{array}$ & $\begin{array}{l}\text { Flower } \\
\text { head diamt } \\
\text { (cm) }\end{array}$ \\
\hline Cco & 120.73 & 75.73 & 73.52 & 6.30 & 3.11 & 7.13 & 46.68 & 7.34 \\
\hline $\mathrm{Co}_{1}(\mathbf{9})$ & 58.12 & 51.00 & 45.49 & 6.18 & 4.05 & 11.61 & 40.14 & 5.38 \\
\hline $\mathrm{Co}_{2}(5)$ & 60.53 & 63.26 & 58.85 & 5.19 & 3.16 & 9.50 & 44.00 & 7.87 \\
\hline $\mathrm{Co}_{3}(1)$ & 54.10 & 43.99 & 35.28 & 5.98 & 3.38 & 10.50 & 22.52 & 3.79 \\
\hline $\mathrm{Co}_{3}(59)$ & 60.73 & 47.23 & 39.85 & 6.17 & 3.43 & 10.67 & 29.84 & 6.20 \\
\hline $\mathrm{Co}_{3}(63)$ & 64.35 & 60.24 & 53.90 & 6.40 & 4.53 & 10.69 & 25.86 & 6.45 \\
\hline $\mathrm{Co}_{4}(1)$ & 58.68 & 64.14 & 54.52 & 4.16 & 3.29 & 9.75 & 24.19 & 4.53 \\
\hline $\mathrm{Co}_{4}(67)$ & 74.67 & 64.00 & 53.19 & 5.11 & 3.28 & 10.79 & 24.54 & 4.40 \\
\hline $\mathrm{Co}_{4}(88)$ & 80.32 & 64.83 & 54.69 & 5.84 & 3.88 & 11.97 & 23.44 & 4.44 \\
\hline $\mathrm{Co}_{5}(55)$ & 68.08 & 62.68 & 54.79 & 5.19 & 2.87 & 11.57 & 30.89 & 7.42 \\
\hline $\mathrm{Co}_{7}(16)$ & 55.17 & 54.87 & 60.33 & 5.58 & 4.49 & 12.03 & 25.70 & 6.40 \\
\hline $\mathrm{Co}_{7}(88)$ & 56.47 & 53.99 & 54.51 & 5.67 & 4.77 & 10.32 & 21.14 & 5.51 \\
\hline $\mathrm{Co}_{8}(5)$ & 65.42 & 59.32 & 55.23 & 5.76 & 2.75 & 11.43 & 28.52 & 5.61 \\
\hline $\mathrm{Co}_{8}(11)$ & 68.35 & 58.22 & 54.23 & 6.36 & 3.44 & 11.85 & 37.50 & 6.31 \\
\hline $\mathrm{Co}_{10}(24)$ & 44.63 & 45.45 & 43.67 & 4.31 & 3.20 & 8.28 & 25.01 & 5.23 \\
\hline $\mathrm{Co}_{10}(26)$ & 47.31 & 48.41 & 47.47 & 5.45 & 3.43 & 8.13 & 29.90 & 6.04 \\
\hline $\mathrm{Co}_{11}(56)$ & 57.41 & 52.86 & 39.37 & 4.34 & 2.32 & 8.45 & 20.79 & 6.18 \\
\hline $\mathrm{Co}_{11}(131)$ & 60.28 & 56.37 & 54.04 & 6.29 & 5.45 & 12.27 & 22.00 & 6.70 \\
\hline $\mathrm{Co}_{12}(3)$ & 53.96 & 65.10 & 55.75 & 3.46 & 2.60 & 7.85 & 21.11 & 4.54 \\
\hline $\mathrm{Co}_{12}(\mathbf{9 7})$ & 53.11 & 66.42 & 55.39 & 4.36 & 3.25 & 8.14 & 22.67 & 5.30 \\
\hline $\mathrm{Co}_{13}(7)$ & 50.51 & 54.87 & 52.64 & 5.09 & 2.41 & 10.60 & 21.75 & 5.59 \\
\hline $\mathrm{Co}_{13}(23)$ & 42.90 & 53.97 & 50.62 & 4.36 & 3.63 & 11.10 & 34.47 & 6.27 \\
\hline $\mathrm{Co}_{13}(45)$ & 44.27 & 51.34 & 49.17 & 4.47 & 3.29 & 11.43 & 33.14 & 5.29 \\
\hline GM & 60.87 & 57.31 & 52.02 & 5.30 & 3.47 & 10.26 & 28.51 & 5.77 \\
\hline S.Em. \pm & 0.70 & 0.80 & 0.75 & 0.08 & 0.08 & 0.12 & 0.49 & 0.10 \\
\hline CD at $5 \%$ & 2.01 & 2.28 & 2.14 & 0.24 & 0.23 & 0.35 & 1.42 & 0.28 \\
\hline
\end{tabular}


Table.2 Phenotypic characterization of $C$. coronarium and its different mutants

\begin{tabular}{|c|c|c|c|c|c|c|c|c|c|}
\hline Species & $\begin{array}{l}\text { Flower } \\
\text { disc } \\
\text { diamt } \\
(\mathrm{cm})\end{array}$ & $\begin{array}{l}\text { Number } \\
\text { of ray } \\
\text { florets }\end{array}$ & $\begin{array}{l}\text { Number of } \\
\text { disc florets }\end{array}$ & $\begin{array}{l}\text { Flower } \\
\text { head } \\
\text { weight } \\
\text { (gm) }\end{array}$ & $\begin{array}{l}\text { Ray } \\
\text { floret } \\
\text { weight } \\
\text { (mg) }\end{array}$ & $\begin{array}{l}\text { Disc } \\
\text { floret } \\
\text { weight } \\
\text { (mg) }\end{array}$ & $\begin{array}{l}\text { Ray floret } \\
\text { length } \\
\text { (cm) }\end{array}$ & $\begin{array}{l}\text { Ray floret } \\
\text { width } \\
\text { (cm) }\end{array}$ & $\begin{array}{l}\text { Flower } \\
\text { head } \\
\text { height } \\
(\mathrm{cm})\end{array}$ \\
\hline Cco & 3.65 & 26.50 & 472.10 & 3.61 & 15.55 & 2.34 & 3.29 & 0.87 & 2.59 \\
\hline $\mathrm{Co}_{1}(\mathbf{9})$ & 1.47 & 275.43 & 5.08 & 2.30 & 6.20 & 0.91 & 1.66 & 0.82 & 3.74 \\
\hline $\mathrm{Co}_{2}(5)$ & 2.31 & 67.03 & 207.11 & 4.58 & 34.81 & 1.71 & 2.60 & 0.94 & 0.85 \\
\hline $\mathrm{Co}_{3}(\mathbf{1})$ & 0.14 & 282.75 & 8.82 & 3.28 & 12.21 & 0.92 & 1.46 & 0.63 & 3.59 \\
\hline $\mathrm{Co}_{3}(59)$ & 0.23 & 405.59 & 14.82 & 4.31 & 14.96 & 0.90 & 1.54 & 0.88 & 4.05 \\
\hline $\mathrm{Co}_{3}(63)$ & 0.26 & 453.41 & 13.30 & 4.13 & 15.24 & 1.17 & 1.82 & 0.96 & 4.23 \\
\hline $\mathrm{Co}_{4}(1)$ & 1.60 & 221.70 & 70.37 & 3.14 & 9.81 & 1.12 & 1.62 & 1.18 & 0.81 \\
\hline $\mathrm{Co}_{4}(67)$ & 1.87 & 144.21 & 73.25 & 3.84 & 8.79 & 1.17 & 1.50 & 0.98 & 2.16 \\
\hline $\mathrm{Co}_{4}(88)$ & 1.65 & 169.50 & 73.09 & 3.50 & 8.89 & 1.51 & 1.55 & 1.24 & 2.37 \\
\hline $\mathrm{Co}_{5}(55)$ & 2.50 & 156.73 & 270.17 & 2.33 & 14.69 & 1.34 & 3.47 & 1.43 & 2.66 \\
\hline $\mathrm{Co}_{7}(16)$ & 1.84 & 141.14 & 151.11 & 3.17 & 15.73 & 1.87 & 1.88 & 0.77 & 2.44 \\
\hline $\mathrm{Co}_{7}(88)$ & 2.22 & 128.14 & 9.92 & 1.88 & 12.59 & 1.19 & 1.72 & 0.82 & 2.26 \\
\hline $\mathrm{Co}_{8}(5)$ & 1.50 & 133.92 & 147.97 & 2.62 & 11.46 & 0.89 & 1.83 & 0.53 & 3.51 \\
\hline $\mathrm{Co}_{8}(11)$ & 1.64 & 138.98 & 157.96 & 3.36 & 12.52 & 0.93 & 1.80 & 0.93 & 3.54 \\
\hline $\mathrm{Co}_{10}(24)$ & 3.36 & 74.52 & 304.40 & 2.78 & 10.52 & 0.87 & 1.62 & 1.10 & 2.11 \\
\hline $\mathrm{Co}_{10}(26)$ & 3.59 & 168.15 & 438.23 & 3.14 & 12.25 & 0.90 & 1.73 & 1.29 & 2.70 \\
\hline $\mathrm{Co}_{11}(56)$ & 1.81 & 178.53 & 224.88 & 4.96 & 16.00 & 1.84 & 2.15 & 1.34 & 3.47 \\
\hline $\mathrm{Co}_{11}(131)$ & 2.67 & 135.47 & 10.80 & 2.66 & 13.37 & 1.40 & 1.87 & 0.89 & 2.65 \\
\hline $\mathrm{Co}_{12}(3)$ & 0.44 & 306.58 & 50.54 & 2.81 & 4.92 & 0.93 & 1.55 & 0.85 & 3.31 \\
\hline $\mathrm{Co}_{12}(\mathbf{9 7})$ & 0.44 & 369.98 & 62.58 & 3.22 & 6.11 & 0.93 & 1.77 & 0.95 & 3.57 \\
\hline $\mathrm{Co}_{13}(7)$ & 0.61 & 266.71 & 30.30 & 1.93 & 6.57 & 0.95 & 1.73 & 0.93 & 4.23 \\
\hline $\mathrm{Co}_{13}(23)$ & 0.61 & 316.25 & 30.68 & 2.27 & 6.52 & 0.94 & 1.48 & 0.81 & 3.89 \\
\hline $\mathrm{Co}_{13}(45)$ & 0.56 & 311.31 & 34.19 & 2.54 & 6.42 & 0.96 & 1.74 & 0.91 & 4.15 \\
\hline GM & 1.60 & 211.84 & 124.42 & 3.14 & 12.00 & 1.20 & 1.88 & 0.95 & 2.99 \\
\hline S.Em. \pm & 0.04 & 2.85 & 2.45 & 0.04 & 0.29 & 0.02 & 0.04 & 0.01 & 0.04 \\
\hline CD at $5 \%$ & 0.12 & 8.15 & 7.00 & 0.13 & 0.83 & 0.07 & 0.11 & 0.04 & 0.11 \\
\hline
\end{tabular}


Table.3 Phenotypic characterization of $C$. segetum and its different mutants

\begin{tabular}{|c|c|c|c|c|c|c|c|c|}
\hline Species & $\begin{array}{l}\text { Plant } \\
\text { height } \\
(\mathrm{cm})\end{array}$ & $\begin{array}{l}\text { Plant } \\
\text { spread (E- } \\
\text { W) }(\mathrm{cm})\end{array}$ & $\begin{array}{l}\text { Plant spread } \\
(\mathrm{N}-\mathrm{S})(\mathrm{cm})\end{array}$ & $\begin{array}{l}\text { Leaf length } \\
(\mathrm{cm})\end{array}$ & $\begin{array}{l}\text { Leaf } \\
\text { width } \\
(\mathrm{cm})\end{array}$ & $\begin{array}{l}\text { Leaf } \\
\text { area } \\
\left(\mathrm{cm}^{2}\right)\end{array}$ & $\begin{array}{l}\text { No of } \\
\text { flowers } \\
\text { /plant }\end{array}$ & $\begin{array}{l}\text { Flower } \\
\text { head } \\
\text { diamt } \\
(\mathrm{cm})\end{array}$ \\
\hline Cse & 75.59 & 66.62 & 65.40 & 6.95 & 3.68 & 11.82 & 61.51 & 6.24 \\
\hline$S_{1-5}$ & 55.33 & 53.00 & 50.26 & 5.45 & 3.45 & 11.73 & 46.92 & 5.26 \\
\hline$S_{1-14}$ & 56.32 & 54.55 & 53.36 & 6.25 & 3.32 & 11.41 & 65.73 & 5.54 \\
\hline$S_{4-6}$ & 42.86 & 40.71 & 37.38 & 5.13 & 2.20 & 6.38 & 43.19 & 6.84 \\
\hline$S_{4-9}$ & 45.99 & 42.88 & 38.28 & 5.22 & 2.34 & 7.15 & 42.88 & 7.10 \\
\hline$S_{5-4}$ & 51.40 & 43.74 & 39.36 & 4.76 & 3.11 & 8.03 & 41.85 & 6.19 \\
\hline GM & 54.58 & 50.24 & 47.34 & 5.62 & 3.01 & 9.42 & 50.34 & 6.19 \\
\hline S.Em. \pm & 0.86 & 1.14 & 1.23 & 0.20 & 0.07 & 0.38 & 1.02 & 0.14 \\
\hline CD at $5 \%$ & 2.71 & 3.61 & 3.89 & 0.65 & 0.24 & 1.22 & 3.22 & 0.46 \\
\hline
\end{tabular}

Table.4 Phenotypic characterization of $C$. segetum and its different mutants

\begin{tabular}{|c|l|l|l|l|l|l|l|l|l|}
\hline Species & $\begin{array}{l}\text { Flower } \\
\text { disc } \\
\text { diamt } \\
\text { (cm) }\end{array}$ & $\begin{array}{l}\text { Number } \\
\text { of ray } \\
\text { florets }\end{array}$ & $\begin{array}{l}\text { Number of } \\
\text { disc florets }\end{array}$ & $\begin{array}{l}\text { Flower } \\
\text { head } \\
\text { weight } \\
\text { (gm) }\end{array}$ & $\begin{array}{l}\text { Ray } \\
\text { floret } \\
\text { weight } \\
\text { (mg) }\end{array}$ & $\begin{array}{l}\text { Disc } \\
\text { floret } \\
\text { weight } \\
\text { (mg) }\end{array}$ & $\begin{array}{l}\text { Ray } \\
\text { floret } \\
\text { length } \\
\text { (cm) }\end{array}$ & $\begin{array}{l}\text { Ray } \\
\text { floret } \\
\text { width } \\
\text { (cm) }\end{array}$ & $\begin{array}{l}\text { Flower } \\
\text { head } \\
\text { height } \\
\text { (cm) }\end{array}$ \\
\hline Cse & 2.43 & 22.77 & 338.76 & 2.19 & 25.43 & 2.03 & 3.13 & 1.40 & 1.96 \\
\hline $\mathbf{S}_{\mathbf{1 - 5}}$ & 1.82 & 20.52 & 358.73 & 2.36 & 24.07 & 1.55 & 1.98 & 0.78 & 2.19 \\
\hline $\mathbf{S}_{\mathbf{1 - 1 4}}$ & 2.13 & 21.03 & 365.21 & 2.32 & 24.97 & 1.63 & 2.28 & 0.86 & 2.40 \\
\hline $\mathbf{S}_{\mathbf{4 - 6}}$ & 2.41 & 20.89 & 472.05 & 2.19 & 25.46 & 2.18 & 2.21 & 0.64 & 2.04 \\
\hline $\mathbf{S}_{\mathbf{4 - 9}}$ & 2.21 & 18.97 & 457.42 & 2.33 & 24.88 & 2.30 & 2.13 & 0.64 & 2.31 \\
\hline $\mathbf{S}_{\mathbf{5 - 4}}$ & 2.07 & 16.10 & 351.70 & 1.95 & 23.55 & 1.43 & 2.11 & 0.90 & 1.96 \\
\hline $\mathbf{G M}_{\mathbf{G}}$ & 2.17 & 20.04 & 390.64 & 2.22 & 24.72 & 1.85 & 2.30 & 0.86 & 2.14 \\
\hline $\mathbf{S . E m . \pm}$ & 0.05 & 0.54 & 8.23 & 0.05 & 1.19 & 0.11 & 0.08 & 0.01 & 0.08 \\
\hline $\mathbf{C D}$ at & 0.17 & 1.72 & 25.93 & 0.18 & 3.77 & 0.35 & 0.25 & 0.05 & 0.26 \\
\hline $\mathbf{5 \%}$ & & & & & & & & \\
\hline
\end{tabular}


Table.5 Phenotypic characterization of $C$. carinatum and its different mutants

\begin{tabular}{|c|c|c|c|c|c|c|c|c|}
\hline Species & $\begin{array}{l}\text { Plant } \\
\text { height } \\
(\mathrm{cm})\end{array}$ & $\begin{array}{l}\text { Plant } \\
\text { spread (E- } \\
\text { W) }(\mathrm{cm})\end{array}$ & $\begin{array}{l}\text { Plant spread } \\
(\mathrm{N}-\mathrm{S})(\mathrm{cm})\end{array}$ & $\begin{array}{l}\text { Leaf length } \\
(\mathrm{cm})\end{array}$ & $\begin{array}{l}\text { Leaf } \\
\text { width } \\
\text { (cm) }\end{array}$ & $\begin{array}{l}\text { Leaf } \\
\text { area } \\
\left(\mathrm{cm}^{2}\right)\end{array}$ & $\begin{array}{l}\text { No of } \\
\text { flowers } \\
\text { /plant }\end{array}$ & $\begin{array}{l}\text { Flower } \\
\text { head } \\
\text { diamt } \\
(\mathrm{cm})\end{array}$ \\
\hline Cca & 115.11 & 65.43 & 48.72 & 7.45 & 4.54 & 14.99 & 43.60 & 8.51 \\
\hline $\mathrm{C}_{9-1}$ & 45.32 & 26.51 & 20.51 & 3.51 & 2.12 & 7.07 & 23.81 & 4.69 \\
\hline$C_{13-1}$ & 40.89 & 27.89 & 22.96 & 5.90 & 4.91 & 21.37 & 15.22 & 6.07 \\
\hline $\mathrm{C}_{15-1}$ & 55.40 & 32.62 & 27.74 & 3.10 & 1.15 & 3.11 & 18.67 & 4.69 \\
\hline GM & 64.17 & 38.11 & 29.98 & 4.90 & 3.18 & 11.63 & 25.32 & 5.99 \\
\hline S.Em. \pm & 5.25 & 1.79 & 1.46 & 0.22 & 0.19 & 0.49 & 0.33 & 0.21 \\
\hline CD at $5 \%$ & 18.16 & 6.20 & 5.06 & 0.78 & 0.69 & 1.69 & 1.16 & 0.72 \\
\hline
\end{tabular}

Table.6 Phenotypic characterization of $C$. carinatum and its different mutants

\begin{tabular}{|c|c|c|c|c|c|c|c|c|c|}
\hline Species & $\begin{array}{l}\text { Flower } \\
\text { disc } \\
\text { diamt } \\
(\mathrm{cm})\end{array}$ & $\begin{array}{l}\text { Number } \\
\text { of ray } \\
\text { florets }\end{array}$ & $\begin{array}{l}\text { Number of } \\
\text { disc florets }\end{array}$ & $\begin{array}{l}\text { Flower } \\
\text { head } \\
\text { weight } \\
\text { (gm) }\end{array}$ & $\begin{array}{l}\text { Ray } \\
\text { floret } \\
\text { weight } \\
\text { (mg) }\end{array}$ & $\begin{array}{l}\text { Disc } \\
\text { floret } \\
\text { weight } \\
\text { (mg) }\end{array}$ & $\begin{array}{l}\text { Ray } \\
\text { floret } \\
\text { length } \\
(\mathrm{cm})\end{array}$ & $\begin{array}{l}\text { Ray } \\
\text { floret } \\
\text { width } \\
\text { (cm) }\end{array}$ & $\begin{array}{l}\text { Flower } \\
\text { head } \\
\text { height } \\
(\mathrm{cm})\end{array}$ \\
\hline Cca & 3.43 & 24.62 & 344.26 & 3.62 & 20.51 & 2.84 & 3.19 & 1.24 & 2.77 \\
\hline $\mathrm{C}_{9-1}$ & 1.03 & 46.30 & 156.90 & 2.92 & 15.07 & 0.38 & 1.90 & 0.81 & 2.27 \\
\hline$C_{13-1}$ & 1.07 & 233.74 & 145.67 & 3.85 & 20.23 & 1.37 & 2.51 & 0.67 & 3.77 \\
\hline$C_{15-1}$ & 1.18 & 16.89 & 244.51 & 2.16 & 21.62 & 0.97 & 2.06 & 0.85 & 2.29 \\
\hline GM & 1.67 & 80.38 & 222.83 & 3.13 & 19.35 & 1.38 & 2.41 & 0.89 & 2.77 \\
\hline S.Em. \pm & 0.04 & 1.69 & 7.36 & 0.10 & 0.6 & 0.20 & 0.08 & 0.06 & 0.08 \\
\hline $\begin{array}{c}\text { CD at } \\
5 \%\end{array}$ & 0.14 & 5.85 & 25.45 & 0.37 & 2.08 & 0.69 & 0.27 & 0.21 & 0.28 \\
\hline
\end{tabular}


Figure.1 Phenotypic characterization of $C$. coronarium and its different mutants

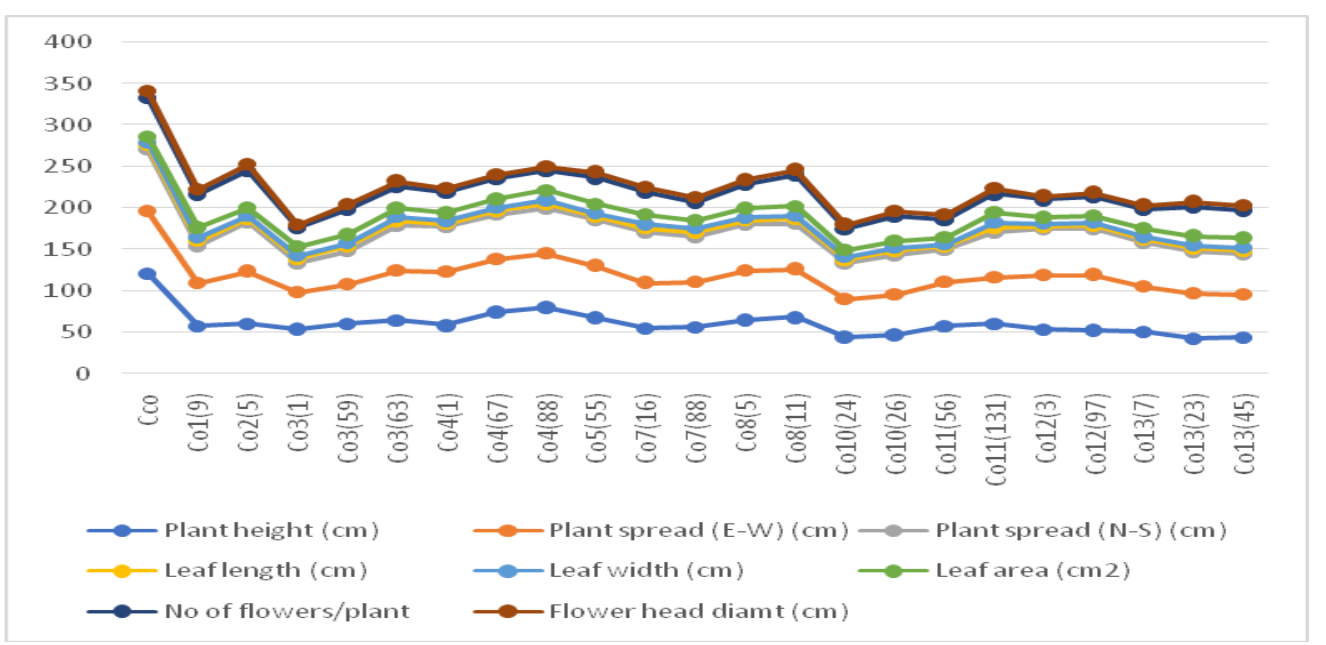

Figure.2 Phenotypic characterization of $C$. coronarium and its different mutants

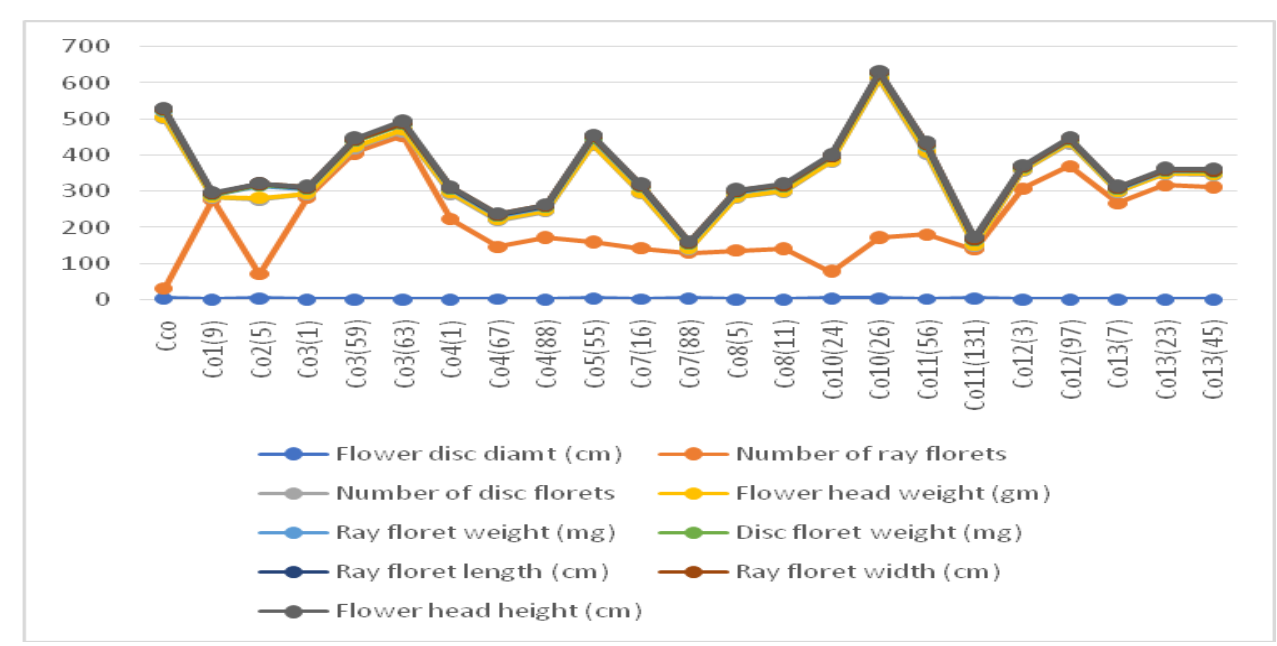


Figure.3 Phenotypic characterization of $C$. segetum and its different mutants

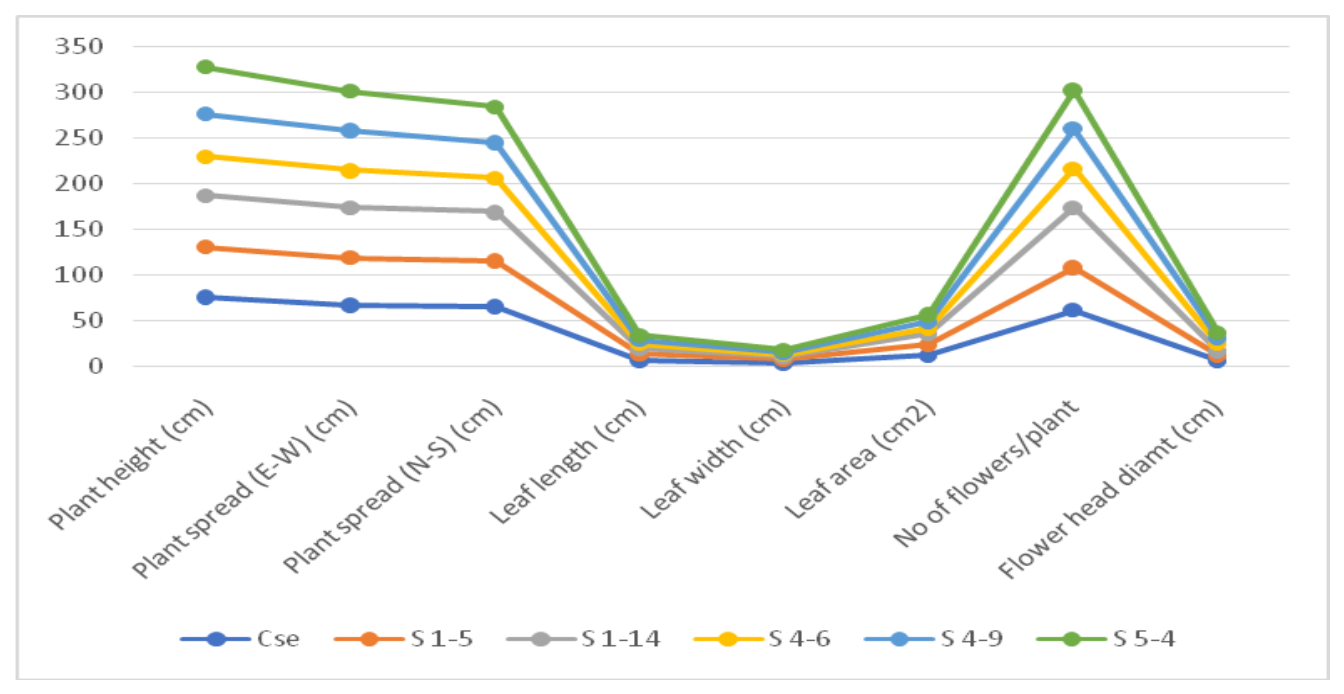

Figure.4 Phenotypic characterization of $C$. segetum and its different mutants

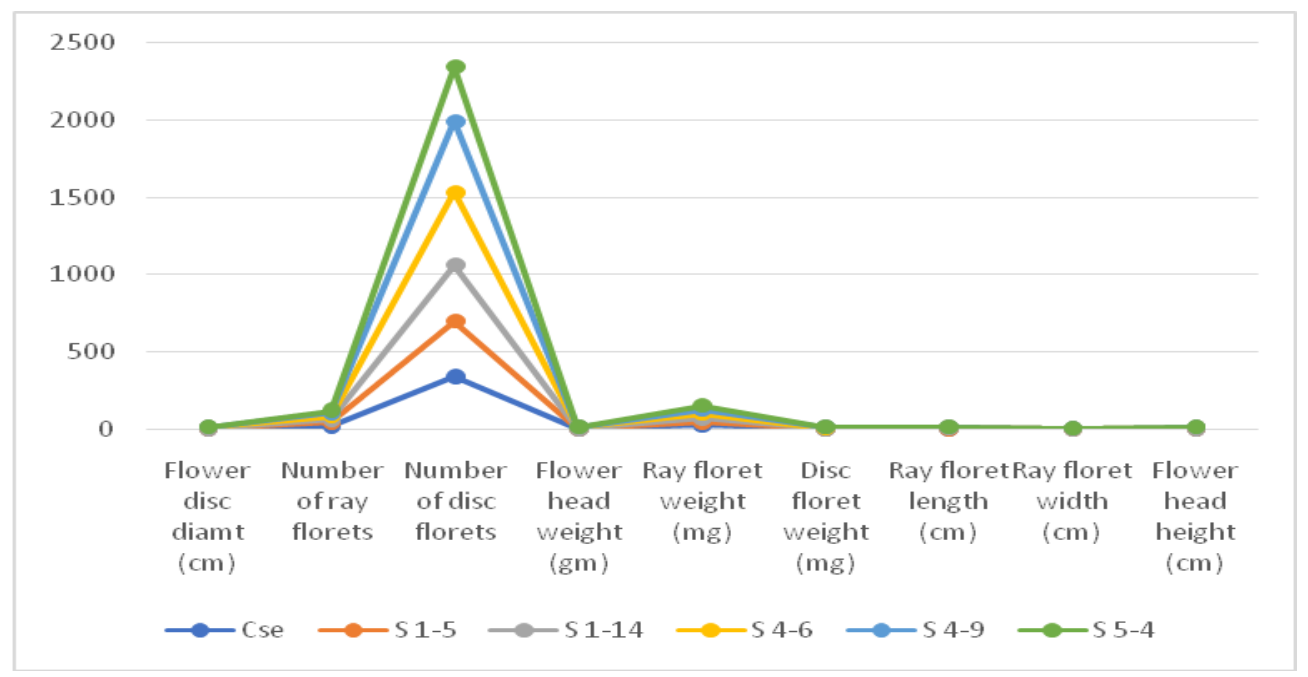


Figure.5 Phenotypic characterization of $C$. carinatum and its different mutants

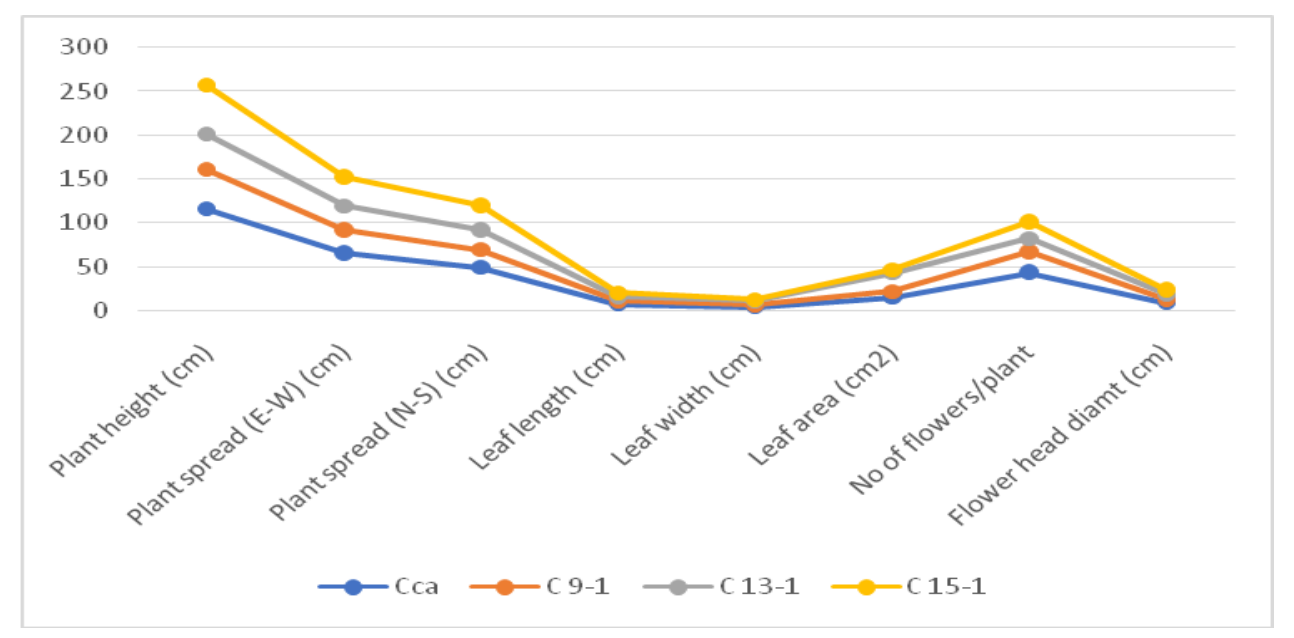

Figure.6 Phenotypic characterization of $C$. carinatum and its different mutants

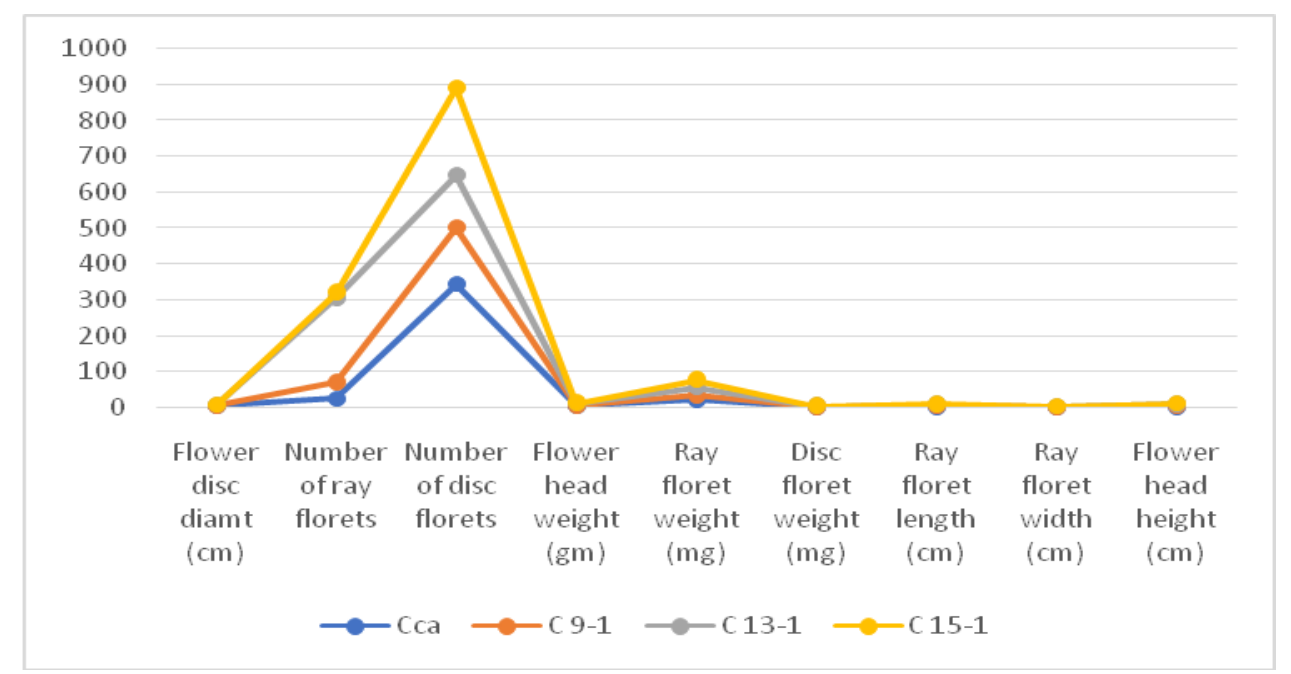


Figure.7 Selected mutants from different species of annual Chrysanthemum

$$
\mathrm{Co}_{2-5}
$$

Co $5-55$

$$
\text { Co } 11-131
$$

$\mathrm{C}_{13-1}$
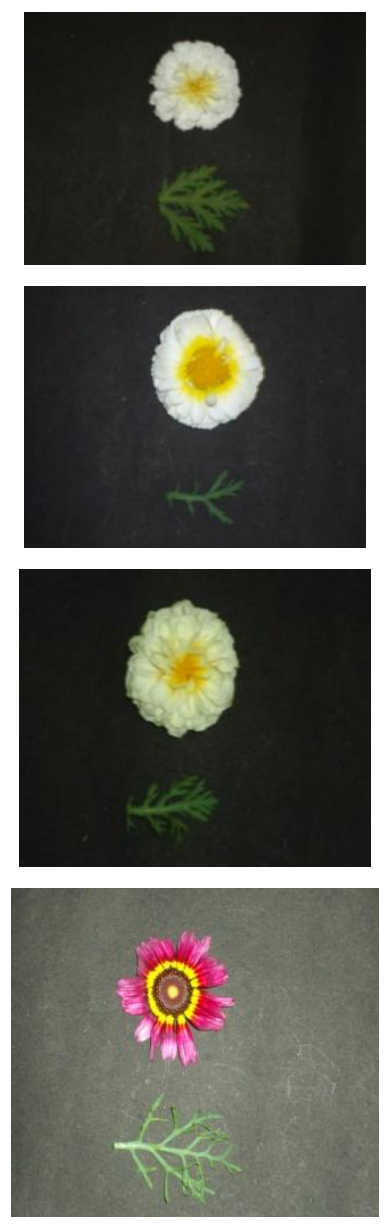
Mutant $\mathrm{S}_{4-9}$ observed a maximum disc floret weight $(2.30 \mathrm{mg})$ which was statistically at par with mutant $\mathrm{S}_{4-6}(2.18 \mathrm{mg})$ and species Cse $(2.03 \mathrm{mg})$ and significantly higher than otherswhile, minimum disc floret weight was found in mutant $\mathrm{S}_{5-4}(1.43 \mathrm{mg})$.Mutant $\mathrm{S}_{1}$ 140bserved maximum flower head height $(2.40 \mathrm{~cm})$ which was statistically at par with mutant $\mathrm{S}_{4-9}(2.31 \mathrm{~cm})$ and significantly higher than others while minimum flower head height was found inmutant $\mathrm{S}_{5-4}$ and species Cse $(1.96 \mathrm{~cm})$.

\section{Chrysanthemum carinatum}

Pursual of data presented in Table 5 and 6 represented that there is a significant variation of different quantitative characteristics of species Chrysanthemum carinatum $(\mathrm{Cca})$ and its all mutants. Findings revealed that plant height $(115.11 \mathrm{~cm})$, plant spread $(\mathrm{E}-\mathrm{W})$ $(65.43 \mathrm{~cm})$ and $(\mathrm{N}-\mathrm{S})(48.72 \mathrm{~cm})$, flower disc diameter $(3.43 \mathrm{~cm})$, leaf length $(7.45 \mathrm{~cm})$, ray floret length $(3.19 \mathrm{~cm})$, width $(1.24 \mathrm{~cm})$ and number of flowers per plant (43.60 no) (Fig 5) were found maximum in species $C$. carinatum (Cca) while, mutant $\mathrm{C}_{13-1}$ had minimum plant height $(40.89 \mathrm{~cm})$ and number of flowers/plant (15.22 no), similar findings were obtained by Gupta and Jugran (1978) in Chrysanthemum as they recorded that significant reduction in plant height, number of flower heads per plant and flower head diameter in the treated plants over control.

Lesser plant spread $(\mathrm{E}-\mathrm{W})(26.51 \mathrm{~cm})$ and $(\mathrm{N}-$ S) $(20.51 \mathrm{~cm})$ were noted in mutantC 9-1 $_{1}$ Datta and Gupta (1980) observed significant reduction in plant spread in Chrysanthemum. Similarly, Banerji and Datta (1990 and 1992) and Zargar et al., (1998) also recorded significantly lesser number of branches and plant spread. Mutant $\mathrm{C}_{13-1}$ had greater leaf width $(4.91 \mathrm{~cm})$, leaf area $\left(21.37 \mathrm{~cm}^{2}\right)$ and number of ray florets (233.74 no) however, minimum leaf width $(1.15 \mathrm{~cm})$, leaf area $(3.11$ $\mathrm{cm}^{2}$ ), flower head diameter $(4.69 \mathrm{~cm})$ and number of ray florets (16.89 no), flower head weight $(2.16 \mathrm{~g})$ were observed in mutant $\mathrm{C}_{15}$ 1.Findings revealed that ray floret weight $(21.62 \mathrm{mg})$ was found more in mutant $\mathrm{C}_{15}$ 1 which was statistically at par with species Cca $(20.51 \mathrm{mg})$ and mutant $\mathrm{C}_{13-1}(20.23 \mathrm{mg})$ (Fig 6) while, minimum ray floret weight was found in mutant $\mathrm{C}_{9-1}(15.07 \mathrm{mg})$. These finding also recorded by Furuya (1998) in Chrysanthemum.

In conclusion, findings revealed that in case of species Chrysanthemum coronarium, under vegetative characteristics, mutant Co 11${ }_{131}$ gave the best results as it had maximum leaf area and leaf width whereas, under flowering characteristics, mutant $\mathrm{Co}_{2-5}$ and mutant Co 5-55 revealed good response.

In species Chrysanthemum segetum, most of the mutants like mutants $\mathrm{S}_{4-9,} \mathrm{~S}_{4-6}, \mathrm{~S}_{1-14}, \mathrm{~S}_{1-}$ ${ }_{5}$ gave good response with regards to flowering characteristics. In the species Chrysanthemum carinatum, mutant C 13-1 showed the best results with respect to vegetative and flowering characters as it had maximum leaf width, leaf area, number of ray florets, flower head weight and flower head height. So, we can use mutants $\mathrm{Co}$ 11-131, $\mathrm{Co}_{2-}$ ${ }_{5}$, Co ${ }_{5-55}$ and ${ }_{13-1}$ for future study.

\section{References}

Anderson, N. O.; Ascher, P. D. and Widmer, R. E. 1992. Inbreeding depression in garden and glasshouse Chrysanthemum $s$ : germination and survivorship. Euphytica, 62: 155-169.

Anonymous. (2008). World news in floriculture. Flora Cul. Intl., 14:16.

Banerji, B. K. and Datta, S. K. (1990). Induction of somatic mutation in Chrysanthemum cv. 'Anupam'. J. Nuclear Agric. Biol., 19: 252-256.

Banerji, B. K. and Datta, S. K. (1992). Gamma rays induced flower shape 
mutation in Chrysanthemum cv. 'Jaya'. J. Nuclear Agric. Biol.21: 73-79.

Banerji, B. K. and Datta, S. K. (2002). Induction and analysis of gamma rayinduced flower head shape mutation in 'Lalima'

Chrysanthemum (Chrysanthemum morifolium). Indian J. Agril. Sci., 5:7-11.

Barakat, M. N.; Abdel-Fattah, R. S.; Badr, M. and El-Torky, M. G. 2010. In vitro mutagenesis and identification of new variants via RAPD markers for improving Chrysanthemum morifolium. African Journal of Agricultural Research,5(8): 748-757.

Datta, S. K. and Gupta, M. N. (1980). Effect of gamma irradiation on rooted cuttings of small flower Chrysanthemum . New Botanist, 7: 73-85.

Datta, S.K. (1988). Chrysanthemum cultivars evolved by induced mutation at National Botanical research Institute, Lucknow, India, Chrysanthemum , 49: $72-75$.

Furuya, H. 1998. Mutation line characteristics of spray type Chrysanthemum irradiated with gamma rays. Bull. Hiroshima Prefectural Agric. Res. Center, 66:3340.

Gaul, H. 1970. Plant injury and lethality. In: Manual on Mutation Breeding. Vienna,
FAO/IAEA. 85-90p.

Gupta, M. N. and Jugran, H. M. 1978. Mutation breeding of Chrysanthemum II. Detection of gamma ray induced somatic mutation in VM2. J. Nuclear Agric. Biol., 7: 50-59.

Hajra, P. K.; Rao, R. R.; Singh, D. K. and Uniyal, B. P. 1995. Flora of India. Vol $12 \& 13$, Asteraceae. Calcutta, BSI.

Misra, P.; Banerji, B. K. and Kumari, A. 2009. Effect of gamma irradiation on Chrysanthemum cultivar 'Pooja' with particular reference to induction of somatic mutation in flower colour and form. J. Orn. Hort., 12: 213-216.

Stevens, P. F. 2001. Angiosperm Phylogeny Website.

Zagorski, J. S.; Ascher, P. D. and Widmer, R. E. 1983. Multigenic self-incompatibility in hexaploid Chrysanthemum . Euphytica, 32:1-7.

Zargar, G. H., Singh, H. C. P., Zeerak, N. A., Wani, S. A. and John, A. Q. 1998. Gamma ray induced somatic mutations in Chrysanthemum . In: Seminar on exploring the Chrysanthemum in India, Lucknow, 4-5 December 1998, National Conference on Chrysanthemum : Proceedings. Lucknow, NBRI, pp. 105110.

\section{How to cite this article:}

Renu and Shant Lal. 2019. Phenotypic Characterization of Mutants from Different Species of Annual Chrysanthemum. Int.J.Curr.Microbiol.App.Sci. 8(09): 2983-2996. doi: https://doi.org/10.20546/ijcmas.2019.809.342 